\title{
Alimentação de alevinos de peixe-rei (Odontesthes bonariensis) com dietas naturais e artificiais ${ }^{1}$
}

\author{
Feeding pejerrey (Odontesthes bonariensis) fingerlings with natural and artificial diets
}

\author{
Sérgio Renato Noguez Piedras ${ }^{2}$ Juvêncio Luís Osório Fernandes Pouey ${ }^{3}$
}

\section{RESUMO}

Neste trabalho, avaliou-se a resposta de alevinos de peixe-rei a diferentes tipos de alimentação, sendo os animais distribuídos num experimento com três tratamentos e quatro repetições, completamente ao acaso. Cada unidade de observação era composta por um aquário de 20 litros contendo 20 animais com três dias de idade. Durante seis semanas foram administradas, três vezes ao dia, as seguintes dietas: $T 1=$ ração farelada, $T 2=$ ração farelada + zooplâncton e T3= zooplâncton . Os resultados mostraram que a presença de zooplâncton resulta em maior crescimento e sobrevivência dos alevinos. Os peixes alimentados apenas com ração farelada apresentaram sobrevivência e crescimento significativamente menores, mas aceitáveis, considerando o estado atual dos estudos de nutrição para o peixe-rei.

Palavras-chave: peixe-rei, zooplâncton, dieta, Odontesthes.

\section{ABSTRACT}

An experiment was run to evaluate three feeding methods for pejerrey fingerlings, in a complete randomized design with four replications. Each experimental unit consisted of a $20 \mathrm{~L}$ aquarium and 20 three days old fingerlings. For six weeks, three times a day, fish received the following diets: $T 1=$ mash diet; $T 2=$ mash diet + zooplankton; $T 3=$ zooplankton. Results showed that zooplankton promotes better fingerlings growth and survival. Fish fed with mash diet showed significantly lower growth and survival, but acceptable considering actual status of nutrition studies for pejerrey.

Key words: pejerrey, zooplankton, ration, Odontesthes.

\section{INTRODUÇÃO}

O sucesso no cultivo de larvas e/ou alevinos de peixes está diretamente relacionado ao fornecimento de alimentação de qualidade e em quantidade suficientes às necessidades específicas de cada espécie. Aimportância do alimento natural é destacada por diversos autores. Para
CASTAGNOLLI\& CYRINO(1986), o hábito alimentardos peixes normalmente se define no estádio adulto, mas o plâncton é alimento indispensável para todas as formas jovens. WATANABE et al. (1983) eUYS \& HECHT (1985) afirmam que o zooplâncton é a principal fonte de alimento na fase de larvicultura, sendo que os rotíferos perfazem 90\% da dieta de larvas da maioria das espécies de peixes.

A alimentação de larvas é considerada uma das fases mais difíceis da aqüicultura (NRC, 1993), pois os organismos estão na fase de diferenciação estrutural e funcional do sistema digestório, o qual na maioria das espécies, passa da alimentação endógena (vitelo) para a alimentação exógena. Por isto, a primeira alimentação apresenta dificuldades de adaptação, sendo recomendado o uso de alimento vivo, ao menos em parte da dieta. A transição do alimento vivo para dieta artificial deve ser gradual, pois, nesta fase, as larvas apresentam o trato digestório imaturo, e as dietas fornecidas podem apresentar-se de difícil digestão. Por outro lado, o alimento natural apresenta determinadas enzimas, hormônios e outros reguladores e fatores de crescimento não encontrados nos alimentos artificiais.

Estudos sobre a alimentação natural são recomendados por CRISPIM et al. (1999), pois apesar das técnicas de alimentação artificial estarem bem desenvolvidas, algumas espécies de peixes apresentam taxas de crescimento mais elevadas na natureza do que em viveiros, quando alimentados artificialmente. PESSOA \& KLEIN (1999) destacam a importância dos rotíferos como alimento básico para larvas de praticamente todos os organismos aquáticos.

Emrelaçãoaopeixe-rei, SAMPAIO \&MINILLO (1995) fazem referência à alimentação artificial de larvas do peixe-rei marinho (Odontesthes argentinensis) e sugerem

${ }^{1} \mathrm{O}$ trabalho é parte da Tese de Doutorado do primeiro autor. Curso de Pós-graduação em Zootecnia, Departamento de Zootecnia, Faculdade de Agronomia Eliseu Maciel (FAEM), Universidade Federal de Pelotas (UFpel). Financiamento da FAPERGS.

${ }^{2}$ Oceonógrafo, Professor Doutor, Universidade Católica de Pelotas. E-mail: sergiopiedras@ibest.com.br

${ }^{3}$ Médico Veterinário, Professor Doutor, Departamento de Zootecnia, FAEM, UFpel, CP 354, 96001-970, Pelotas, RS. Bolsista do

CNPq. juvencio@ufpel.tche.br 
uma taxa de arraçoamento de $25 \%$ da biomassa para maior crescimento e sobrevivência. ALT \& MAÇADA (1996) compararam a eficiência do alimento vivo (Artemia salina) a uma ração rica em proteína vegetal e uma ração rica em proteína animal no peixe-rei (Odontesthes sp.) do estuário da Lagoa dos Patos e concluíram que o alimento vivo proporcionou melhor desempenho, seguido da ração com proteína animal. ESCALANTE (2001) afirma ser o peixe-rei (Odontesthes bonariensis) uma espécie essencialmente zooplanctófaga, mas com versatilidade anatômica que lhe permite ampliar seu espectro trófico, buscando alimento em outras comunidades, incluindo a possibilidade de aceitar alimentos artificiais.

RADÜNZ NETO (2003) alerta para o fato de que o uso de alimento vivo apresenta alguns inconvenientes, como custos de produção em laboratório, variabilidade na produção em função das condições climáticas, quando cultivados em tanques de terra e riscos de introdução de predadores e patógenos nos sistemas de criação. Com relação ao alimento artificial, salienta que este apresenta maior facilidade de aquisição e estocagem, além da uniformidade dos ingredientes e agilidade no seu fornecimento. $\mathrm{O}$ autor afirma ainda que as pesquisas em relação à nutrição de larvas estão sendo desenvolvidas no sentido de limitar o emprego de alimento vivo aos primeiros dias de vida, substituindo-o por alimentos totalmente artificiais.

O objetivo deste trabalho foi verificar a possibilidade de produzir alevinos de peixe-rei alimentandoos com uma dieta artificial.

\section{MATERIAL E MÉTODOS}

O experimento foi desenvolvido em um sistema composto de 12 aquários, com capacidade de 20 litros cada um, e equipados com filtros biológicos e sistemas de aeração independentes. Em cada aquário, foram colocados 20 animais com três dias de idade, medindo $9,0 \mathrm{~mm}$ de comprimento total e $3,8 \mathrm{mg}$ de peso médio. Para manter o equilíbrio eletrolítico dos peixes e como medida preventiva ao surgimento de patologias, a água dos aquários foi mantida com salinidade entre 2 e $3 \%$ através da adição de $\mathrm{NaCl}$. A qualidade da água foi monitorada três vezes por semana, controlando-se os níveis de oxigênio dissolvido, temperatura, $\mathrm{pH}$, condutividade, alcalinidade, gás carbônico, amônia e nitrito, seguindo metodologia recomendada por APHA (1998).

Durante seis semanas, os peixes foram submetidos a três tipos de alimentação, sendo o Tratamento um (T1) ração comercial farelada, o Tratamento dois (T2) ração farelada e zooplâncton e o Tratamento três (T3) zooplâncton. Foi utilizada uma ração farelada comercial com uma composição básica de: minhoca desidratada, farinha de peixe, farinha de vísceras, farinha de carne e ossos, farelo de arroz, farinha de soja, farelo de soja integral, óleo vegetal, cloreto de sódio e premix vitamínico mineral. O zooplâncton foi produzido em tanques de terra a partir de adubação química. Durante os primeiros 10 dias, foi fornecido exclusivamente rotíferos vivos (Keratella sp.) e, após este período, o zooplâncton oferecido vivo era constituído de $90 \%$ de cladóceros (Daphnia sp.) e 10\% de copépodos (Calanoida). A alimentação foi fornecida três vezes ao dia: às 8,12 e 18 horas, ad libitum.

As características nutricionais da ração e do zooplâncton foram determinadas pelo "Esquema de Weende" no Laboratório de Nutrição Animal do Departamento de Zootecnia da Faculdade de Agronomia Eliseu Maciel - Universidade Federal de Pelotas. O delineamento experimental utilizado foi completamente ao acaso, com três tratamentos e quatro repetições. Os resultados foram analisados através do efeito das dietas sobre o crescimento em comprimento total e peso final e sobrevivência dos alevinos. Os dados obtidos ao final do experimento foram submetidos à análise de variância (ANOVA) e as médias dos tratamentos comparados pelo teste de Tukey, usando o Software SAS 6.12 (1998), considerando-se como diferença significativa entre as médias quando $\mathrm{P} \leq 0,05$.

\section{RESULTADOS E DISCUSSÃO}

As características físicas e químicas da água monitoradas durante o período experimental não apresentaram variações significativas entre os tratamentos e mantiveram-se em amplitudes semelhantes aos recursos hídricos regionais e, portanto, aceitáveis para a espécie estudada. A temperatura média foi de $21,1^{\circ} \mathrm{C}$, o oxigênio dissolvido foi de $5,7 \mathrm{mg} \mathrm{L}^{-1}, \mathrm{pH} 8,0$, gás carbônico $0,82 \mathrm{mg} \mathrm{L}^{-1}$, alcalinidade $67,1 \mathrm{mg} \mathrm{L}^{-1} \mathrm{de}$ $\mathrm{CaCo}_{3}$, amônia total $0,016 \mathrm{mg} \mathrm{L}^{-1}$ e nitrito $0,14 \mathrm{mg} \mathrm{L}^{-1}$.

Os resultados das análises bromatológicas da ração comercial e do zooplâncton, ingredientes utilizados nas dietas ministradas aos alevinos, estão apresentados na tabela 1. Estes resultados são semelhantes aos descritos por PIEDRAS \& POUEY (2000), que sugerem uma dieta artificial com 50\% de proteína bruta e $3600 \mathrm{kcal}$ de energia digestível para alevinos de peixe-rei. Já os resultados registrados para o zooplâncton são semelhantes aos obtidos por CORREIA (1998) em relação a crustáceos em geral, utilizados na alimentação de camarões.

A tabela 2 mostra que os peixes alimentados com as dietas T2 (ração farelada + zooplâncton) e T3 (zooplâncton) apresentaram crescimento e 
Tabela 1 - Características nutricionais da dieta artificial e do zooplâncton utilizados no experimento.

\begin{tabular}{|c|c|c|c|c|}
\hline \multirow[b]{2}{*}{ Características } & \multicolumn{2}{|c|}{ Ração } & \multicolumn{2}{|c|}{ Zooplâncton } \\
\hline & Matéria natural & Matéria seca & Matéria natural & Matéria seca \\
\hline Matéria seca (\%) & 88,93 & -- & 5,76 & -- \\
\hline Proteína bruta $(\%)$ & 45,05 & 50,66 & 3,66 & 63,59 \\
\hline Cinzas $(\%)$ & 11,29 & 12,70 & 1,14 & 19,79 \\
\hline Extrato etéreo $(\%)$ & 11,01 & 12,38 & 0,24 & 4,10 \\
\hline Fibra bruta (\%) & 3,57 & 4,02 & 0,44 & 7,71 \\
\hline Energia $\left(\mathrm{kcal} \mathrm{kg}^{-1}\right)$ & 3.673 & -- & 4.466 & -- \\
\hline
\end{tabular}

Tabela 2 - Resultados finais de crescimento em peso, comprimento total e sobrevivência ao final do experimento

\begin{tabular}{|c|c|c|c|c|}
\hline Parâmetros & T1 (ração) & T2 (ração+zooplâncton) & T3 (zooplâncton) & C.V. (\%) \\
\hline Peso médio (mg) & $31,7 \pm 6,2^{b}$ & $171,3 \pm 7,5^{\mathrm{a}}$ & $153,5 \pm 6,8^{a}$ & 31,23 \\
\hline Comprimento médio $(\mathrm{mm})$ & $10,7 b \pm 0,04$ & $31,0 \mathrm{a} \pm 0,05$ & $29,1 \mathrm{a} \pm 0,05$ & 10,58 \\
\hline Sobrevivência média (\%) & $38 b \pm 1,6$ & $77 \mathrm{a} \pm 2,0$ & $79 \mathrm{a} \pm 1,8$ & 14,83 \\
\hline
\end{tabular}

Letras diferentes nas linhas indicam haver diferença significativa entre as médias dos tratamentos, através do teste de Tukey, considerando $\mathrm{P}$ $\leq 0,05$

sobrevivência significativamente maiores do que T1 (Ração farelada), o que mostra a eficiência do zooplâncton na alimentação do peixe-rei. Este resultado está de acordo com RADÜNZ NETO (2003), quando cita que o sucesso na criação de larvas, da maioria das espécies de peixes brasileiros, depende do fornecimento de alimento natural (principalmente por rotíferos, cladóceros e copépodos), pelo menos nos primeiros dias de vida.

Embora as dietas artificiais venham sendo utilizadas de forma decisiva no cultivo de peixes como fator de sustentabilidade ecológica ou de viabilidade técnico-econômica da atividade (COLDEBELLA \& RADÜNZ NETO, 2002), a maioria dos estudos de nutrição de alevinos de espécies nativas no sul do Brasil são relativos ao jundiá (Rhamdia quelen) e referem-se à formulação de dietas artificiais (ULIANA et al., 2001; FONTINELLI, 1997; COLDEBELLA \& RADÜNZNETO, 2002), não servindo como referencial para o peixe-rei, o que dificulta uma discussão mais abrangente dos resultados aqui registrados.

Por outro lado, resultados como os obtidos por DIAS et al. (1998), que testaram o alimento natural em relação ao alimento artificial e a mistura de ambos, obtiveram o melhor resultado (maior peso médio final e maior sobrevivência) em larvas de pacu (Colossoma mitrei) com o alimento artificial. Entretanto SHARMA \& CHAKRABATI (1999), em experimento semelhante, obtiveram maior sobrevivência e crescimento em larvas de carpa comum (Cyprinus carpio), com o alimento natural, demonstrando, com isto, a especificidade do tema.

\section{CONCLUSÕES}

O alimento vivo proporciona maiores ganhos em crescimento e sobrevivência durante os primeiros 40 dias de cultivo de alevinos de peixe-rei. $\mathrm{O}$ uso de alimento artificial na fase de alevinagem do peixe-rei, embora resulte em crescimento e sobrevivência inferiores ao alimento natural, apresenta-se como uma alternativa que deve ser melhor estudada, tendo em vista as vantagens do seu custo e manejo em cultivos comerciais.

\section{REFERÊNCIAS BIBLIOGRÁFICAS}

APHA. Standard methods for examination of water and wastewater. New York : American Public Health Association, 1998. 824p.

ALT, C.C.; MAÇADA, A.P. Alimentação de larvas de peixerei, Odontesthes sp., cultivadas em laboratório (Pisces: Atherinidae). In: SIMPÓSIO BRASILEIRO DE AQÜICUlTURA, 9., 1996, Sete Lagoas, MG. Resumos... Sete Lagoas : Associação Brasileira de Aqüicultura, 1996. p.73.

CAStagnolli, N.; CYRINo, E. Piscicultura nos trópicos. São Paulo : Manole. 1986. 152p.

COLDEBELLA, I.; RADÜNZ NETO, J. Farelo de soja na alimentação de alevinos de jundiá Rhamdia quelen. Ciência Rural, Santa Maria, v.32, n.3, p.499-503, 2002. 
CORREIA, E.S. Influência da alimentação natural no cultivo semi-intensivo do camarão de água doce Macrobrachium rosenbergii (de Man, 1879). 1998. $136 \mathrm{f}$. Tese (Doutorado em Ecologia e Recursos Naturais) - Curso de Pós-graduação em Ecologia e Recursos Naturais, Universidade Federal de São Carlos.

CRISPIM, M.C.; CAVALHEIRO, J.M.O.; PEREIRA, J.A. A influência do zooplâncton no crescimento de peixes em viveiros de aquacultura. In: CONGRESSO BRASILEIRO DE ENGENHARIA DE PESCA, 11. e CONGRESSO LATINOAMERICANO DE ENGENHARIA DE PESCA, 1999, Olinda, PE. Anais... Olinda : Associação dos Engenheiros de Pesca de Pernambuco, 1999. V.1, p.78-87.

DIAS, T.C.R.; CASTAGNOLli, N.; CARNEIRO, D.J. Alimentação de larvas de pacu (Colossoma mitrei Berg, 1895) com dietas naturais e artificiais. In: SIMPÓSIO LATINOAMERICANO, 6. e SIMPÓSIO BRASILEIRO DE AQÜICUlTurA, 5., 1998, Florianópolis, SC. Anais... Florianópolis : Associação Brasileira de Aqüicultura, 1998. V.2. p.500-504.

ESCALANTE, A.H. Alimentación natural del pejerrey. In: GURSMAN, F. Fundamentos biologicos, economicos y sociales para una correcta gestión del recurso pejerrey. Buenos Aires : Imprenta Rivadavia, 2001. Cap.9, p. 64-74.

FONTINELLI, E. Efeitos do uso do concentrado protéico de soja, com e sem suplementação de aminoácidos, sobre o crescimento e sobrevivência das larvas de jundiá Rhamdia quelen. 1997. 38f. Dissertação (Mestrado em Zootecnia) Curso de Pós-graduação em Zootecnia, Universidade Federal de Santa Maria.

NRC. Nacional Research Council (U.S). Committee on Animal Nutrition. 1. Fishes - Nutrition - Requirements. 2. Fishes - Feedings and feeds. Washington, DC. : National Academy of Sciences, 1993. 114p.

PESSOA, E.V.; KLEIN, V.L.M Análise do valor proteico do rotífero Brachionus plicatilis - submetido à diferentes dietas. In: CONGRESSO BRASILEIRO DE ENGENHARIA DE PESCA, 11. e CONGRESSO LATINO-AMERICANO DE
ENGENHARIA DE PESCA, 1., 1999, Olinda, PE. Anais... Olinda : Associação dos Engenheiros de Pesca de Pernambuco, 1999. V.1. p.262- 268.

PIEDRAS, S.R.N.; POUEY, J.L.F.O. Avaliação de quatro dietas balanceadas no crescimento e sobrevivência de larvas de peixe-rei (Odontesthes bonariensis). In: SIMPÓSIO BRASILEIRO DE AQUiCUltura, 5., 2000, Florianópolis, SC. Anais... Florianópolis : Associação Brasileira de Aqüicultura, 2000. CDRoom.

RADÜNZ NETO, J. Alimento natural versus ração balanceada na larvicultura de peixes. Capturado em $07 \mathrm{de}$ ago. 2003. Online. Disponível na Internet http://www.sbz.org.br/ eventos/PortoAlegre/homepagessbz/Radunz.htm.

SAMPAIO, L.A.; MINILLO, A. Alimentação de peixe rei marinho (Odontesthes argentinensis) com diferentes taxas de arraçoamento. In: ENCONTRO SUL BRASILEIRO DE AQÜICULTURA, 3. e ENCONTRO RIOGRANDENSE DE TECNICOS EM AQÜICULTURA, 6., 1995, Porto Alegre, RS. Anais... Porto Alegre : Universidade Federal do Rio Grande do Sul, 1995. V.1. p.34-39.

SAS. Statistical Analysis System Institute. Ed.6.12. Cary: SAS, 1998. CD-Room.

SHARMA, J.G.; CHAKRABATI, R. Larvae rearing of commun carp Cyprinus carpio: A comparision between natural and artificial diets under three stocking densities. Journal of the World Aquaculture Society, Baton Rouge, v.30, n.4, p.490-495, 1999.

ULIANA, O.; DA SILVA, J.H.; RADÜNZ NETO, J. Diferentes fontes de lipídios testados na criação de larvas de jundiá (Rhamdia quelen), Pisces, Pimelodidae. Ciência Rural, Santa Maria, v.31, n.1, p.129-133, 2001.

UYS, W.; HECHT, T. Evaluation and preparation of an optimal dry feed for the primary nursing of Clarias garipineus larvae (Pisces: Claridae). Aquaculture, Amsterdam, n.47, p.175-181, 1985.

WATANABE, T.; KITAJIMA, C.; FUJTTA, S. Nutritional values of live organisms used in Japan for mass propagation of fish: a review. Aquaculture, Amsterdam, v.34, p.115-143, 1983. 